\title{
Discovery of diffuse radio emission in the galaxy cluster A1689
}

\author{
V. Vacca ${ }^{1,2}$, F. Govoni ${ }^{2}$, M. Murgia ${ }^{2}$, G. Giovannini ${ }^{3,4}$, L. Feretti $^{4}$, M. Tugnoli ${ }^{4}$, M. A. Verheijen ${ }^{5}$, and G. B. Taylor ${ }^{6,7}$ \\ 1 Dipartimento di Fisica, Università degli Studi di Cagliari, Cittadella Universitaria, 09042 Monserrato (CA), Italy \\ e-mail: vvacca@oa-cagliari.inaf.it \\ 2 INAF - Osservatorio Astronomico di Cagliari, Strada 54, Loc. Poggio dei Pini, 09012 Capoterra (Ca), Italy \\ 3 Dipartimento di Astronomia, Università degli Studi di Bologna, via Ranzani 1, 40127 Bologna, Italy \\ ${ }^{4}$ INAF - Istituto di Radioastronomia, via P. Gobetti 101, 40129 Bologna, Italy \\ 5 Kapteyn Astronomical Institute, University of Groningen, Landleven 12, Groningen, The Netherlands \\ 6 Department of Physics and Astronomy, University of New Mexico, MSC 07 4220, Albuquerque, New Mexico, USA \\ 7 Adjunct Astronomer at the National Radio Astronomy Observatory, Socorro, NM 87801, USA
}

Received 30 June 2011 / Accepted 13 September 2011

\begin{abstract}
Aims. The aim of this work is to investigate the possible presence of extended diffuse synchrotron radio emission associated with the intracluster medium of the complex galaxy cluster A1689.

Methods. The radio continuum emission of A1689 has been investigated by analyzing archival observations at $1.2 \mathrm{and} 1.4 \mathrm{GHz}$ obtained with the Very Large Array in different configurations.

Results. We report the detection of an extended, diffuse, low-surface brightness radio emission located in the central region of A1689. The surface brightness profile of the diffuse emission at $1.2 \mathrm{GHz}$ indicates a central radio brightness of $\simeq 1.7 \mu \mathrm{Jy} / \operatorname{arcsec}^{2}$ and the $3 \sigma$ radio isophothes reveal the largest linear size to be $730 \mathrm{kpc}$. Given its central location, the low-level surface brightness, and the comparatively large extension, we classify the diffuse cluster-wide emission in A1689 as a small radio halo.
\end{abstract}

Key words. galaxies: clusters: general - galaxies: clusters: individual: A1689 - magnetic fields - large-scale structure of Universe

\section{Introduction}

According to the hierarchical scenario of structure formation, massive galaxy clusters form from the merger of galaxy groups and subclusters. During these mergers, gravitational energies are released that are as high as $\gtrsim 10^{64} \mathrm{erg}$ (e.g., Sarazin 2002). This significant amount of energy drives shocks and turbulence into the thermal intracluster medium (ICM): the energy is injected on large spatial scales and then cascades turbulently to smaller scales. Shocks and the turbulence associated with a major cluster merger event are thought to accelerate particles and compress magnetic field in the ICM (e.g. Roettiger et al. 1999; Skillman et al. 2008; Skillman et al. 2011). These nonthermal components are expected to lead to large-scale diffuse synchrotron emission associated with the ICM - an expectation confirmed by sensitive radio observations. In fact, in an increasing number of merging galaxy clusters, diffuse sources known as radio halos and radio relics, which have no obvious optical counterpart, have been detected (Feretti \& Giovannini 2008; Ferrari et al. 2008).

Radio halos and radio relics are diffuse, low-surfacebrightness $\left(\sim 1 \mu \mathrm{Jy} \operatorname{arcsec}^{-2}\right.$ at $\left.1.4 \mathrm{GHz}\right)$, steep-spectrum ${ }^{1}$ $(\alpha \gtrsim 1)$ synchrotron sources with linear size $\gtrsim \mathrm{Mpc}$. The radio halo emission permeates the central volume of some galaxy clusters, is generally characterized by a regular morphology, and is not polarized. Instead, radio relic emission is located in the galaxy cluster outskirts, usually filamentary, and polarized $(10-30 \%)$. While radio halos are thought to be sustained mainly

\footnotetext{
${ }^{1}$ We use the convention $S_{v} \propto v^{-\alpha}$
}

by turbulence due to cluster mergers (e.g., Brunetti \& Lazarian 2007), radio relics are supposed to trace the shock waves generated during these processes of cluster formation (e.g., Ensslin et al. 1998).

Radio halos offer a unique chance to study the nonthermal components of the ICM in the central region of the clusters on Mpc scales but, unfortunately, their faintness means their statistics remain poor. To date, only about 30 radio halos are known (Giovannini et al. 2009). They are all found in clusters displaying features of intense merger activity (Buote 2001; Feretti 2002; Govoni et al. 2004; Cassano et al. 2010; Rossetti et al. 2011), but not all merging clusters appear to host a radio halo. X-ray and radio properties of the clusters hosting such sources seem to be intimately connected, as indicated by the similarity of the $\mathrm{X}$-ray and radio morphology, by the correlation between the radio halo power and X-ray cluster temperature (Liang et al. 1999; Colafrancesco 1999) and by the strong correlation between the radio halo power $P_{1.4 \mathrm{GHz}}$ at $1.4 \mathrm{GHz}$ and the $\mathrm{X}$-ray cluster luminosity $L_{X}$ (Feretti 2002; Brunetti et al. 2007; Giovannini et al. 2009). Actually, recent deep radio observations have also revealed the presence of radio halos in clusters characterized by low X-ray luminosity (e.g. 0217+70: Brown et al. 2011; A523: Giovannini et al. 2011), a more complex situation than described by the present models and numerical simulations of radio halo formation.

While radio halos are typically found in merging clusters, few relaxed, cool-core, galaxy clusters exhibit signs of diffuse synchrotron emission that extends far from the dominant radio galaxy at the cluster center, forming a so-called mini-halo. 
Burns et al. (1992) was the first to recognize a distinction between cluster-wide halos, such as in Coma, and mini-halos associated with cool cores, such as in Perseus. Mini-halos are extended on a moderate scale (typically $\simeq 500 \mathrm{kpc}$ ) and, in common with the large-scale halos observed in merging clusters of galaxies, have a steep spectrum and very low surface brightness. Their relatively small angular size in combination with, in many cases, strong emission of the central radio galaxy, complicates their detection, thus our current observational knowledge on mini-halos is limited to only a handful of clusters. The origin and physical properties of mini halos are still poorly known (Gitti et al. 2004; Govoni et al. 2009). We note that, although cooling core clusters are generally considered relaxed systems, when analyzed in detail they sometimes reveal peculiar X-ray features in the cluster center, which may indicate a link between the mini-halo emission and some minor merger activity. Indeed, Burns et al. (2008) have simulated the formation of both cool core and noncool core clusters in the same numerical volume. These simulations confirm that non-cool clusters are formed via major mergers early in their history, which destroyed the cool cores and left significant residual kinetic energy in the gas that might be used to power the radio halos. In contrast, cool core clusters do not suffer any major mergers, thus preserving the central cool regions. However, the cool core clusters do experience regular smaller mergers, which still inject energy into the ICM, but more modestly than in the non-cool core clusters. These minor mergers might power the mini-halos.

Further studies are required to better understand extended diffuse synchrotron radio sources and their connection with the dynamical state of the cluster. In this context, as a part of an ongoing program aimed at finding new diffuse sources in galaxy clusters, we investigate the radio emission of the galaxy cluster A1689.

In this work we present the detection of a diffuse synchrotron radio source in this complex galaxy cluster. In Sect. 2 we summarize the cluster properties. In Sect. 3 we describe the radio observation and the data reduction. In Sect. 4 we show the results and we attempt to classify this diffuse radio emission. Finally, in Sect. 5 we draw some conclusions.

Throughout this paper we adopt a $\Lambda \mathrm{CDM}$ cosmology with $H_{0}=71 \mathrm{~km} \mathrm{~s}^{-1} \mathrm{Mpc}^{-1}, \Omega_{\mathrm{m}}=0.27$, and $\Omega_{\Lambda}=0.73$. At the distance of $\operatorname{A1689}(z=0.1832$; Struble \& Rood 1999), the luminosity distance is $D_{\mathrm{L}}=880 \mathrm{Mpc}$, and $1^{\prime \prime}$ corresponds to $3.05 \mathrm{kpc}$.

\section{A1689 properties}

A1689 is a galaxy cluster characterized by a complex optical and X-ray structure. At first inspection, it has a regular spherical shape and a strong peak in the surface brightness profile in X-rays (Peres et al. 1998), features that apparently indicate a cooling core. Indeed, Chen et al. (2007) found a short central cooling time for A1689 $t_{\text {cool }}=4.7 \pm 0.4 \mathrm{Gyr}$ and a high accretion rate $\dot{M}=683_{-182}^{239} M_{\odot} \mathrm{yr}^{-1}$. Its Einstein radius is the largest known so far (Tyson et al. 1990; Miralda-Escudé \& Babul 1995; Clowe \& Schneider 2001; Broadhurst et al. 2005a; Broadhurst et al. 2005b; Umetsu \& Broadhurst 2008), a characteristic that has made it attractive for lensing analysis. Strong and weak lensing studies have revealed discrepant mass values. Differences in the mass estimations have also been found between lensing and $\mathrm{X}$-ray observations.

By means of XMM-Newton observations, Andersson \& Madejski (2004) found evidence of an ongoing merger. They found a nonuniform radial temperature of the X-ray emitting gas across the cluster, and these features suggest a complex dynamical state. A major merger in which a subcluster moves along the line-of-sight could also explain the different mass estimates from X-ray and lensing analysis. An alternative explanation of this difference has been given by Lemze et al. (2008). By using a model-independent approach based on a simultaneous fit of lensing and X-ray surface brightness data, they find good agreement between the lensing mass profile and the X-ray emission profile. Their model, however, implies a temperature discrepancy with respect to the one derived solely from X-ray observations. The authors state that their result could support the presence of colder, denser, and more luminous small-scale structures that could be responsible for a bias in the observed temperature (Kawahara et al. 2007). Peng et al. (2009) refute this view and show that X-ray and lensing estimates can be made consistent by considering a prolate distribution for the gas temperature and density with the major axis aligned with the line-of-sight.

By using the Hubble's Advanced Camera for Surveys (ACS) and combining the strong and weak lensing approaches, Limousin et al. (2007) apply an accurate mass model to describe the observed substructures, obtaining good agreement between the strong and weak lensing analysis. To reduce the discrepancy between X-ray and lensing approaches, Riemer-Sørenson et al. (2009) combined this lensing analysis with high-resolution X-ray Chandra temperature and hardness ratio images. They find consistent mass profiles and indications that the main clump is in a hydrostatical equilibrium. By means of the same ACS observations, Coe et al. (2010) trace the most detailed mass distribution with a resolution of $25 \mathrm{kpc}$. They estimate a mass $M_{200}=$ $1.8_{-0.3}^{+0.4} \times 10^{15} M_{\odot} h_{70}^{-1}$ within a radius $r_{200}=2.4_{-0.2}^{+0.1} \mathrm{Mpc} h_{70}^{-1}$. In addition, their results agree with the presence of a high mass along the line-of-sight, confirming previous studies present in the literature. Analyzing the same ACS dataset, Leonard et al. (2011) detect two dominant peaks of mass concentration that they identify as possible remnants of a recent merger.

The head-on merger scenario also seems supported by optical observations. In fact, the central region of the cluster lacks a dominant galaxy at its centroid, while it hosts multiple nuclei/galaxies within a single optical halo and possibly merging. Indeed, Girardi et al. (1997) find that A1689 appears to consist of three distinct groups of galaxies possibly aligned and separated well in velocity, suggesting a merger along the line of sight. Such a geometry of the merger leads to an apparent enhancement of the observed velocity dispersion that, by using the temperature measured by White \& Fabian (1995), they find to be $1350 \mathrm{~km} \mathrm{~s}^{-1}$.

\section{Radio observations and data reduction}

We present archival observations of A1689 at 1.2 and $1.4 \mathrm{GHz}$ performed at the Very Large Array (VLA) in spectral line mode in the A, C, and DnC configurations. The details of the observations are summarized in Table 1 . The data were reduced following standard procedures using the NRAO's Astronomical Image Processing System (AIPS) package. Surface brightness images were produced using the AIPS task IMAGR. In the C and DnC configuration the data were collected with a total bandwidth of $12.5 \mathrm{MHz}$, subdivided into seven channels with a bandwith of $\sim 1.6 \mathrm{MHz}$ each. The source $1331+305$ (3C286) was used as flux and bandpass calibrator. The nearby source 1354-021 was observed for complex gain calibration. Radio interference was carefully excised channel by channel, and several cycles of self-calibration and imaging were applied to remove residual 
Table 1. Details of the VLA observations of A1689.

\begin{tabular}{cccccccc}
\hline \hline $\begin{array}{c}\text { RA } \\
(\mathrm{J} 2000)\end{array}$ & $\begin{array}{c}\text { Dec } \\
(\mathrm{J} 2000)\end{array}$ & $\begin{array}{c}\text { Obs. frequency, } v \\
(\mathrm{MHz})\end{array}$ & $\begin{array}{c}\text { Bandwidth } \\
(\mathrm{MHz})\end{array}$ & VLA Configuration & $\begin{array}{c}\text { Time } \\
(\mathrm{h})\end{array}$ & Date & Project \\
\hline 131130.2 & -012010.6 & $1365 / 1435$ & 25.0 & $\mathrm{~A}$ & 3.0 & Mar. 29,30,31 2002 & AI0098 \\
131130.2 & -012035.4 & 1193 & 12.5 & $\mathrm{C}$ & 6.0 & Jan. 13, 1999 & AZ0111 \\
131130.2 & -012035.4 & 1193 & 12.5 & DnC & 7.8 & Feb. 03,05 1999 & AZ0111 \\
\hline
\end{tabular}

Notes. Columns 1, 2: pointing position; Col. 3: observing frequency; Col. 4: observing bandwidth; Col. 5: VLA configuration; Col. 6: time on source; Col. 7: dates of observations; Col. 8: VLA project name.

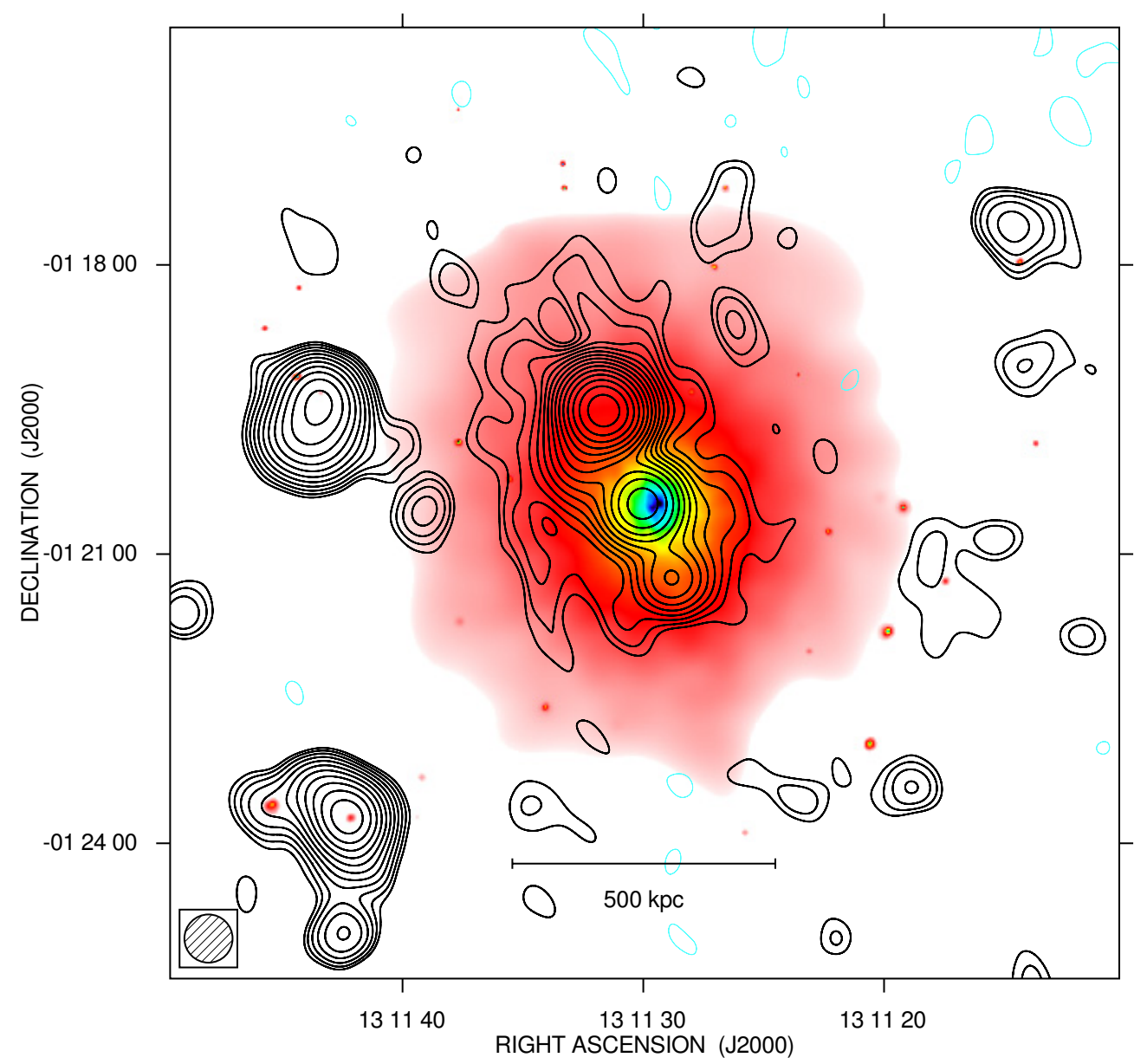

Fig. 1. Total intensity radio contours at $1.2 \mathrm{GHz}$ (VLA in DnC configuration) with an FWHM of $30^{\prime \prime} \times 30^{\prime \prime}$. The contour levels are drawn at $-3 \times 10^{-4} \mathrm{Jy} /$ beam, $3 \times 10^{-4} \mathrm{Jy} /$ beam, and the rest are spaced by a factor $\sqrt{2}$. The sensitivity $(1 \sigma)$ is $1 \times 10^{-4} \mathrm{Jy} /$ beam. The contours of the radio intensity are overlaid on a Chandra X-ray (Obs. id: 6930) $0.1-10 \mathrm{keV}$ band, adaptively smoothed count image.

phase variations. We averaged the seven channels together in the gridding process using IMAGR. The A configuration was calibrated by using the source $1331+305$ as flux calibrator, and the nearby source $1246-075$ as phase reference. The two IFs were averaged to obtain the surface brightness image.

\section{Diffuse emission in A1689}

The radio iso-contours at $1.2 \mathrm{GHz}$ of A1689 are shown in Fig. 1. This image was obtained with the VLA in DnC configuration, and it was convolved with a circular FWHM beam of $30^{\prime \prime}$. To compare the radio and X-ray cluster emission, the radio contours are overlaid on the Chandra image in the $0.1-10 \mathrm{keV}$ band. We find that the central region of A1689 is permeated by a low-surface brightness diffuse emission with a few discrete sources embedded. The radio morphology of the diffuse emission is quite regular with a round shape, following the regular structure of the cluster X-ray emission. As measured from the $3 \sigma$ radio isophote, the overall diffuse emission has an angular extension of about 4' (the Largest Linear Size is $L L S \simeq 730 \mathrm{kpc}$ at the cluster distance). However, as pointed out in Murgia et al. (2009), we note that the size of the diffuse emission calculated from the contour levels should be considered carefully, since it depends on the sensitivity of the radio image.

To separate the diffuse radio emission from discrete sources, we produced images at higher resolution. In Fig. 2 we present the radio contours of A1689 at $1.2 \mathrm{GHz}$ taken with the VLA in C configuration, convolved with an FWHM beam of $20^{\prime \prime}$. The discrete sources are labeled in the figure. A few discrete radio sources (labeled A, B, C) are embedded in the diffuse 

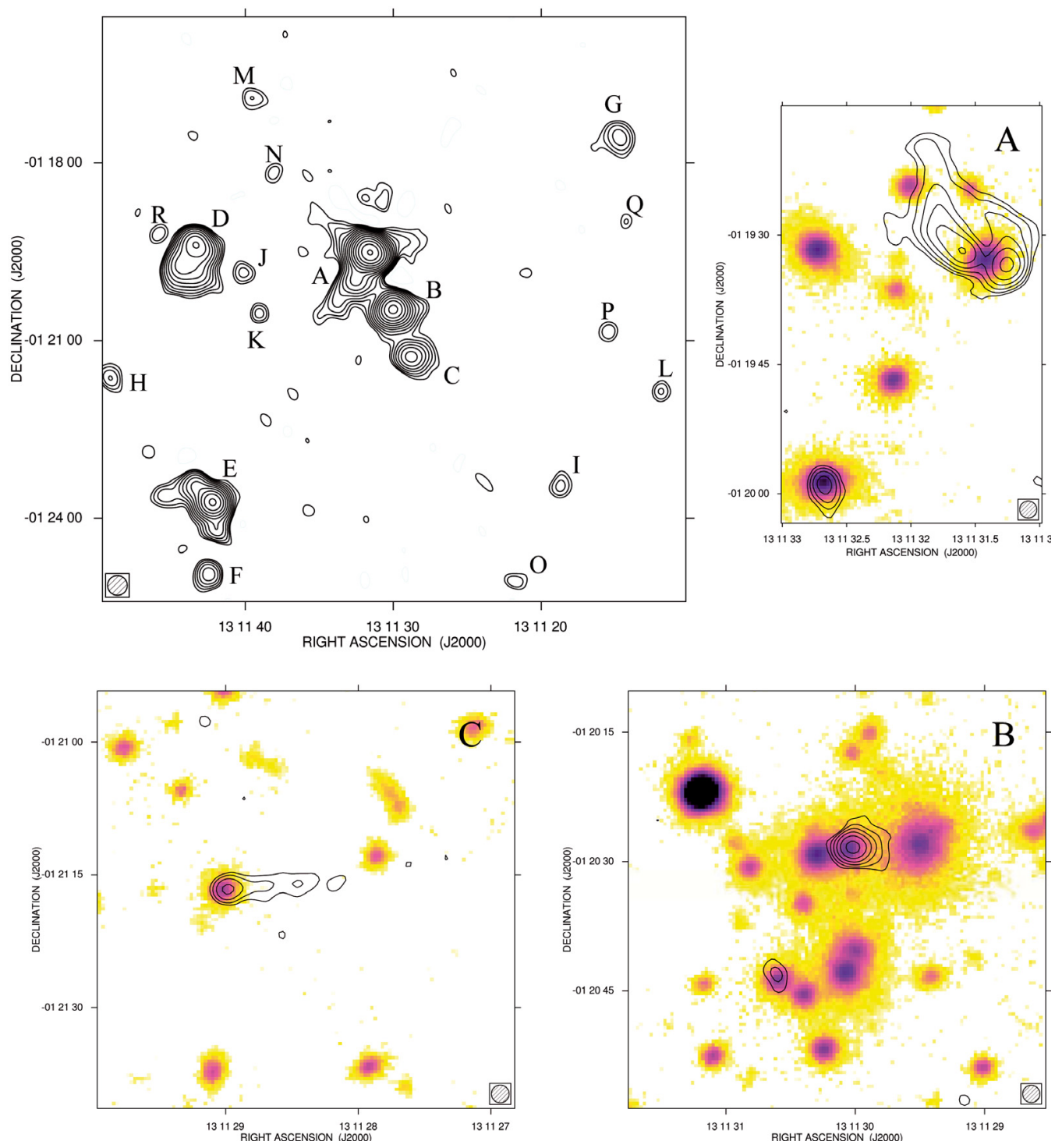

Fig. 2. Top left panel: total intensity radio contours at $1.2 \mathrm{GHz}$ (VLA in C configuration) with an FWHM of $20^{\prime \prime} \times 20^{\prime \prime}$. The contour levels are drawn at $-3.3 \times 10^{-4} \mathrm{Jy} /$ beam, $3.3 \times 10^{-4} \mathrm{Jy} /$ beam, and the rest are spaced by a factor $\sqrt{2}$. The sensitivity $(1 \sigma)$ is $1.1 \times 10^{-4} \mathrm{Jy} / \mathrm{beam}$. Bottom left, right panels: total intensity radio contours at $1.4 \mathrm{GHz}$ (VLA in A configuration) with an FWHM of $2^{\prime \prime} \times 2^{\prime \prime}$. The first contour level is drawn at $13.5 \times 10^{-5} \mathrm{Jy} / \mathrm{beam}$, and the rest are spaced by a factor 2 . The sensitivity $(1 \sigma)$ is $4.5 \times 10^{-5} \mathrm{Jy} / \mathrm{beam}$. The contours of the radio intensity are overlaid on the red plate of the Sloan Digital Sky Survey.

cluster emission, and their positions and flux densities are given in Table 2. Source B is located close to the cluster X-ray peak. In the bottom and righthand panels of Fig. 2, their radio contours at 1.4 GHz taken with the VLA in A configuration (convolved with an FWHM beam of $2^{\prime \prime}$ ) are overlaid to the optical Sloan Digital Sky Survey. From this figure it is evident that source A, when observed at high resolution, is indeed composed of two distinct discrete sources: a wide-angle tailed radio source and a point source, shown respectively in the top right and bottom lefthand corners of the top righthand panel of Fig. 2 (the flux density of source A given in Table 2 includes both sources). All these discrete sources have an optical counterpart.

To ensure that the large-scale diffuse emission is not caused by the blending of discrete sources, in Fig. 3 we present the total intensity radio contours at $1.2 \mathrm{GHz}$ with the VLA in DnC configuration after subtraction of discrete sources. We produced an image of the discrete sources by using only the longest baselines of the DnC configuration dataset and uniform weighting. The
Table 2. Information on discrete radio sources embedded in the central diffuse cluster emission.

\begin{tabular}{lccl}
\hline \hline Label & $\begin{array}{c}\text { RA } \\
(\mathrm{J} 2000)\end{array}$ & $\begin{array}{c}\text { Dec } \\
(\mathrm{J} 2000)\end{array}$ & $\begin{array}{l}S_{1.2 \mathrm{GHz}} \\
(\mathrm{mJy})\end{array}$ \\
\hline $\mathrm{A}$ & 131131.5 & -011931 & $62.0 \pm 2.0$ \\
$\mathrm{~B}$ & 131130.0 & -012028 & $14.0 \pm 0.4$ \\
$\mathrm{C}$ & 131129.0 & -012117 & $3.9 \pm 0.1$ \\
\hline
\end{tabular}

Notes. Columns 1: source label; Cols. 2, 3: Source position (RA, Dec); Col. 4: source flux density at $1.2 \mathrm{GHz}$.

clean components of this image were then subtracted in the $(u, v)$ plane by using the AIPS task UVSUB. The image with the discrete sources subtracted confirms the presence of a low-surface brightness diffuse radio emission at the cluster center. The radio contours are overlaid on the Chandra X-ray image presented in 


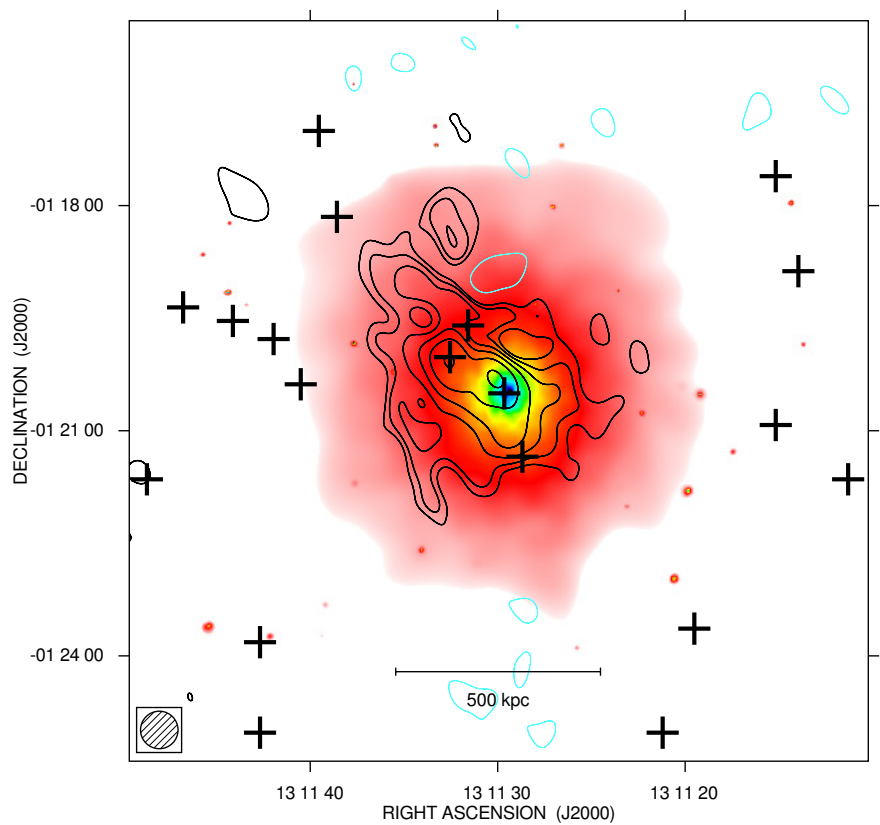

Fig. 3. Total intensity radio contours at $1.2 \mathrm{GHz}$ with the VLA in DnC configuration after subtraction of discrete sources. The image has an FWHM of $30^{\prime \prime} \times 30^{\prime \prime}$. The contour levels are drawn at $-3 \times 10^{-4} \mathrm{Jy} /$ beam, $3 \times 10^{-4} \mathrm{Jy} / \mathrm{beam}$, and the rest are spaced by a factor $\sqrt{2}$. The sensitivity $(1 \sigma)$ is $1 \times 10^{-4} \mathrm{Jy} /$ beam. Crosses indicate the positions of the subtracted discrete sources. The contours of the radio intensity are overlaid on the Chandra X-ray image shown in Fig. 1.

Fig. 1. Crosses indicate the positions of the subtracted discrete sources.

The total flux density is calculated from the DnC dataset after a primary beam correction by integrating the total intensity surface brightness in the region of the diffuse emission down to the $3 \sigma$ level. The resulting total flux density at $1.2 \mathrm{GHz}$ is estimated to be $\simeq(91.6 \pm 2.7) \mathrm{mJy}$. By subtracting the flux density of the embedded discrete sources $\mathrm{A}, \mathrm{B}$, and $\mathrm{C}$ as derived in the C configuration dataset (see Table 2 ), a flux density of $\simeq(11.7 \pm$ 3.4) mJy appears to be associated with the low-brightness diffuse emission. This flux density value corresponds to a radio power of $P_{1.2 \mathrm{GHz}}=1.08 \times 10^{24} \mathrm{~W} \mathrm{~Hz}^{-1}$. To compare this result with studies of other radio halos, we rescaled the corresponding radio power at $1.4 \mathrm{GHz} P_{1.4 \mathrm{GHz}}=9.21 \times 10^{23} \mathrm{~W} \mathrm{~Hz}^{-1}$, correcting by a factor 0.85 as estimated assuming a spectral index $\alpha=1$.

A1689 is part of an X-ray flux-limited galaxy cluster sample selected from the ROSAT All-Sky Survey by Reiprich \& Böhringer (2002). Its X-ray luminosity (corrected for our cosmology) in the $0.1-2.4 \mathrm{keV}$ band is $1.2 \times 10^{45} \mathrm{erg} \mathrm{s}^{-1}$. Therefore, the radio power $P_{1.4 \mathrm{GHz}}$, the radio's largest linear size $(L L S)$, and the X-ray luminosity $\left(L_{\mathrm{X}}\right)$ of A1689 agree with the $P_{1.4 \mathrm{GHz}}-L L S$ and $P_{1.4 \mathrm{GHz}}-L_{\mathrm{X}}$ relations known for the other halos in clusters (Giovannini et al. 2009).

In Fig. 4 we plot the radio power calculated at $1.4 \mathrm{GHz}$ versus the $0.1-2.4 \mathrm{keV} \mathrm{X}$-ray luminosity for clusters hosting radio halos. Classical powerful radio halos in X-ray luminous clusters have been found to show a correlation between the radio power and the X-ray luminosity. A1689 appears consistent with the relation found for other radio halos. The outliers are the few known radio halos over-luminous in radio with respect to the empirical radio - X-ray correlation, revealing a complex scenario for the radio halo formation (Giovannini et al. 2011).

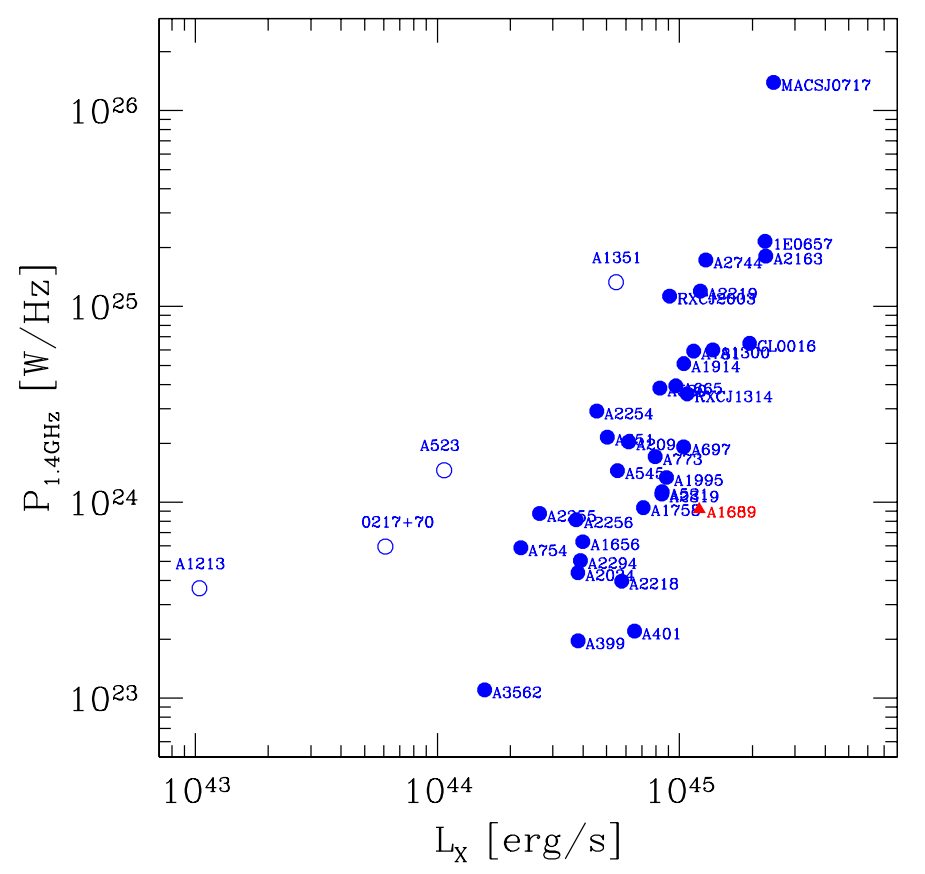

Fig. 4. Relation between the halo radio power at $1.4 \mathrm{GHz}$ and the cluster X-ray luminosity between 0.1 and $2.4 \mathrm{keV}$. A1689 is indicated with a red triangle. Blue dots are classical radio halos, while empty blue dots are outlier clusters. The data are taken from Giovannini et al. (2009) and references therein, with the addition of CL0016+16 (Giovannini \& Feretti 2000), MCSJ0717+3745 (Bonafede et al. 2009), A399 (Murgia et al. 2010), A781 (Govoni et al. 2011), A523 (Giovannini et al. 2011), and 0217+70 (Brown et al. 2011).

\subsection{Azimuthally averaged brightness profile of the radio emission}

In the lefthand panel of Fig. 5 we show the azimuthally averaged radio halo brightness profile at $1.2 \mathrm{GHz}$ obtained from the DnC configuration image after subtracting the discrete sources (see Fig. 3) and correcting for the primary beam attenuation. Each data point represents the average brightness in concentric annuli of half beam width $\left(\simeq 15^{\prime \prime}\right)$ centered on the cluster X-ray peak. The observed brightness profile is traced down to a level of $3 \sigma$.

Following Murgia et al. (2009), we modeled the radio halo brightness profile, $I(r)$, with an exponential of the form $I(r)=$ $I_{0} \mathrm{e}^{-r / r_{\mathrm{e}}}$, whose best-fit is shown in the left panel of Fig. 5. The proposed method for deriving the radio brightness, the length scale, hence the radio emissivity of diffuse sources, is relatively independent of the sensitivity of the radio observation. The exponential model is attractive in its simplicity and involves a minimal set of free parameters. Obviously, it cannot account for the local deviations from the circular symmetry of the diffuse emission.

The fit is performed in the image plane as described in Murgia et al. (2009). To properly take the resolution into account, the exponential model is first calculated in a 2dimensional image, with the same pixel size and field of view as observed, and then convolved with the same beam by means of a fast Fourier transform. Finally, the model is azimuthally averaged with the same set of annuli used to obtain the observed radial profile. All these functions are performed at each step during the fit procedure. As a result, the values of the central brightness, $I_{0}$, and the e-folding radius $r_{\mathrm{e}}$ provided by the fit are deconvolved quantities, and their estimate includes all the uncertainties related to the sampling of the radial profile in annuli of finite width. The fit procedure has been implemented in the 

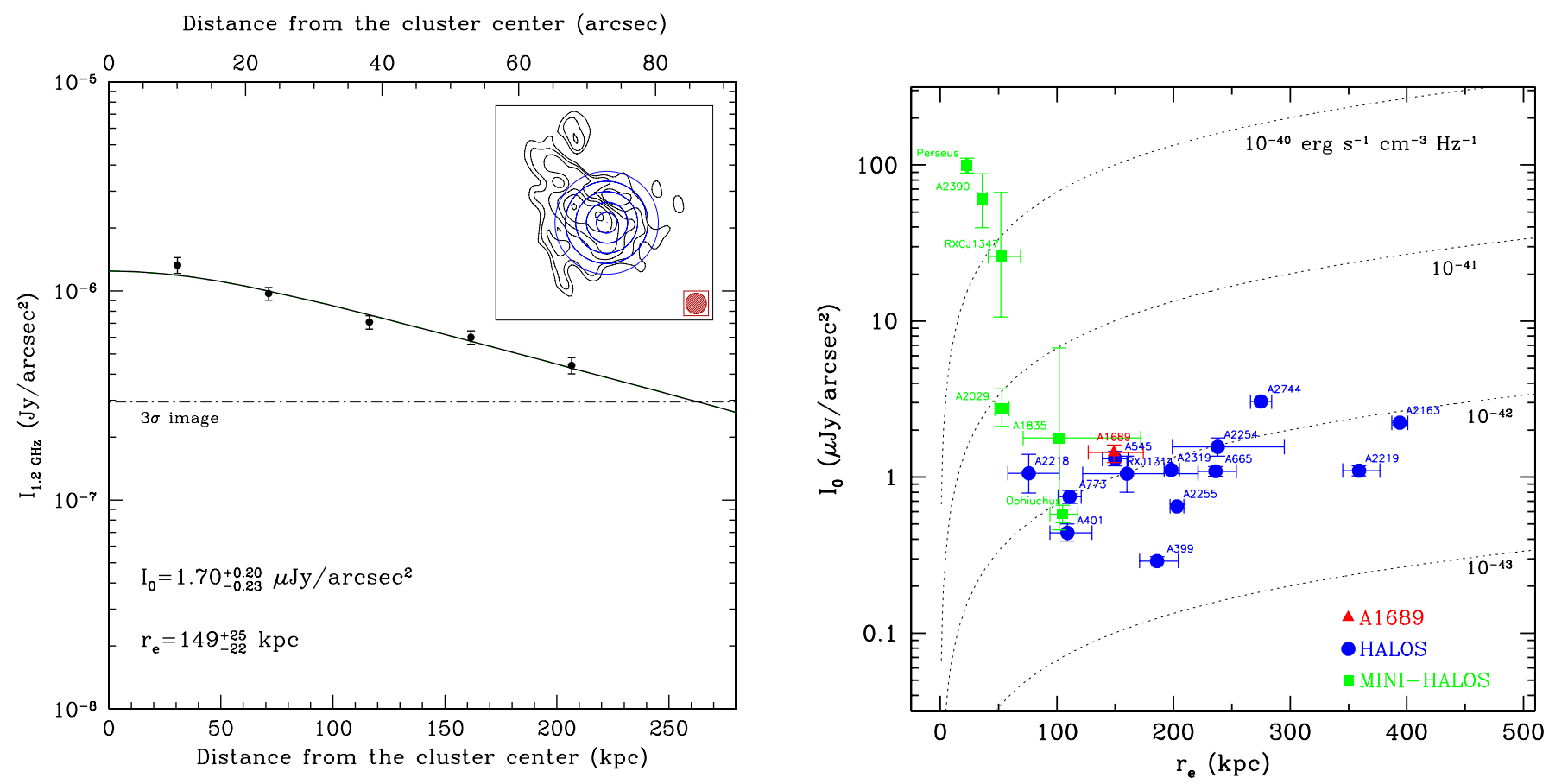

Fig. 5. Left: the azimuthally averaged brightness profile of the radio halo emission in A1689 at $1.2 \mathrm{GHz}$. The profile has been calculated in concentric annuli centered on the cluster X-ray peak, as shown in the inset panel. The horizontal dashed-dotted line indicates the $3 \sigma$ noise level of the radio image, while the continuous line indicates the best-fit profile described by an exponential law (see text). Right: best-fit central brightness $I_{0}$ at $1.4 \mathrm{GHz}$ versus the length scale $r_{\mathrm{e}}$ of A1689 in comparison with azimuthally averaged brightness profiles of radio halos and mini-halos taken from the literature (Murgia et al. 2009, 2010). The dotted lines indicate regions of constant synchrotron emissivity. The central brightness of A1689 has been rescaled at $1.4 \mathrm{GHz}$ with $\alpha=1$ (see text).

software FARADAY (Murgia et al. 2004). The best-fit of the exponential model at $1.2 \mathrm{GHz}$ yields a central brightness ${ }^{2}$ of $I_{0}=\left(1.70_{-0.23}^{+0.20}\right) \mu \mathrm{Jy} / \operatorname{arcsec}^{2}$ and $r_{\mathrm{e}}=\left(149_{-22}^{+25}\right) \mathrm{kpc}$. The average radio emissivity over the volume of a sphere of radius $3 r_{\mathrm{e}}$ is

$\langle J\rangle \simeq 7.7 \times 10^{-41}(1+z)^{3+\alpha} \frac{I_{0}}{r_{\mathrm{e}}}\left(\mathrm{erg} \mathrm{s}^{-1} \mathrm{~cm}^{-3} \mathrm{~Hz}^{-1}\right)$

where $r_{\mathrm{e}}$ and $I_{0}$ are in units of $\mathrm{kpc}$ and $\mu \mathrm{Jy} / \operatorname{arcsec}^{2}$, respectively. From the central brightness and the e-folding radius, we obtained for A1689 an average radio emissivity calculated over the volume of a sphere with radius of $3 r_{\mathrm{e}}$, k-corrected with $\alpha=1$, of $\langle J\rangle=1.7 \times 10^{-42} \mathrm{ergs}^{-1} \mathrm{~cm}^{-3} \mathrm{~Hz}^{-1}$.

In the righthand panel of Fig. 5, we show the best-fit central brightness $I_{0}$ rescaled at $1.4 \mathrm{GHz}$ with $\alpha=1$ versus the length scale $r_{\mathrm{e}}$ of A1689 in comparison with the set of radio halos and mini-halos analyzed in Murgia et al. (2009) and Murgia et al. (2010). As previously pointed out, radio halos can have quite different length scales, but their emissivity is remarkably similar from one halo to another. In contrast, mini-halos span a wide range of radio emissivity. Some of them, like Perseus (Pedlar et al. 1990, Burns et al. 1992), RXJ1347.5-1145 (Gitti et al. 2007) and A2390 (Bacchi et al. 2003) are characterized by a radio emissivity that is more than two orders of magnitude greater than that of radio halos. On the other hand, the mini-halos in cooling core clusters like A2029, Ophiuchus, and A1835 (Govoni et al. 2009) have a radio emissivity that is much more typical of halos in merging clusters rather than to the minihalos previously known. A1689 populates the same region of

\footnotetext{
2 We checked that the fit of the exponential disk performed by masking, and not subtracting, the discrete sources is consistent within the uncertainties with the reported values for $I_{0}$ and $r_{\mathrm{e}}$.
}

the $I_{0}-r_{\mathrm{e}}$ plane of the other radio halos known in the literature. Although the e-folding radius of A1689 is not particularly extended, it is larger than the e-folding radius of the other minihalos (they typically have $r_{\mathrm{e}} \lesssim 100 \mathrm{kpc}$ ). Therefore, the physical properties of the diffuse emission in A1689 seem in good agreement with the extrapolation of the properties of the other radio halos known in the literature.

\subsection{Comparison of thermal and nonthermal emission}

To investigate the possible presence of a radio - X-ray correlation in A1689, in Fig. 6 we present a quantitative comparison between the radio halo emission at $1.2 \mathrm{GHz}$ after the correction for the primary beam attenuation and the Chandra X-ray image in the $0.1-10 \mathrm{keV}$ band. Only regions where the radio halo emission is higher than a level of $3 \sigma$ have been considered. The radial profile was produced by using the same concentric annuli presented in Fig. 5. Each dot represents the average emission in each annulus. By fitting the trend with a power law relation

$I_{1.2 \mathrm{GHz}} \propto I_{\mathrm{X}}^{\mathrm{a}}$

we obtained $\mathrm{a}=0.42 \pm 0.02$ (Fig. 6, left panel), reinforcing the turbulence model of particle acceleration (e.g., Xu et al. 2010). The sublinear slope comes from the radial profile of the radio emission being flatter than the X-ray one, as shown in the righthand panel of Fig. 6, as in Coma and in A2319 (Govoni et al. 2001; Brown \& Rudnick 2011). A similar analysis was performed for the mini-halo in the Ophiucus cluster, where a superlinear correlation has instead been observed (Govoni et al. 2009). 

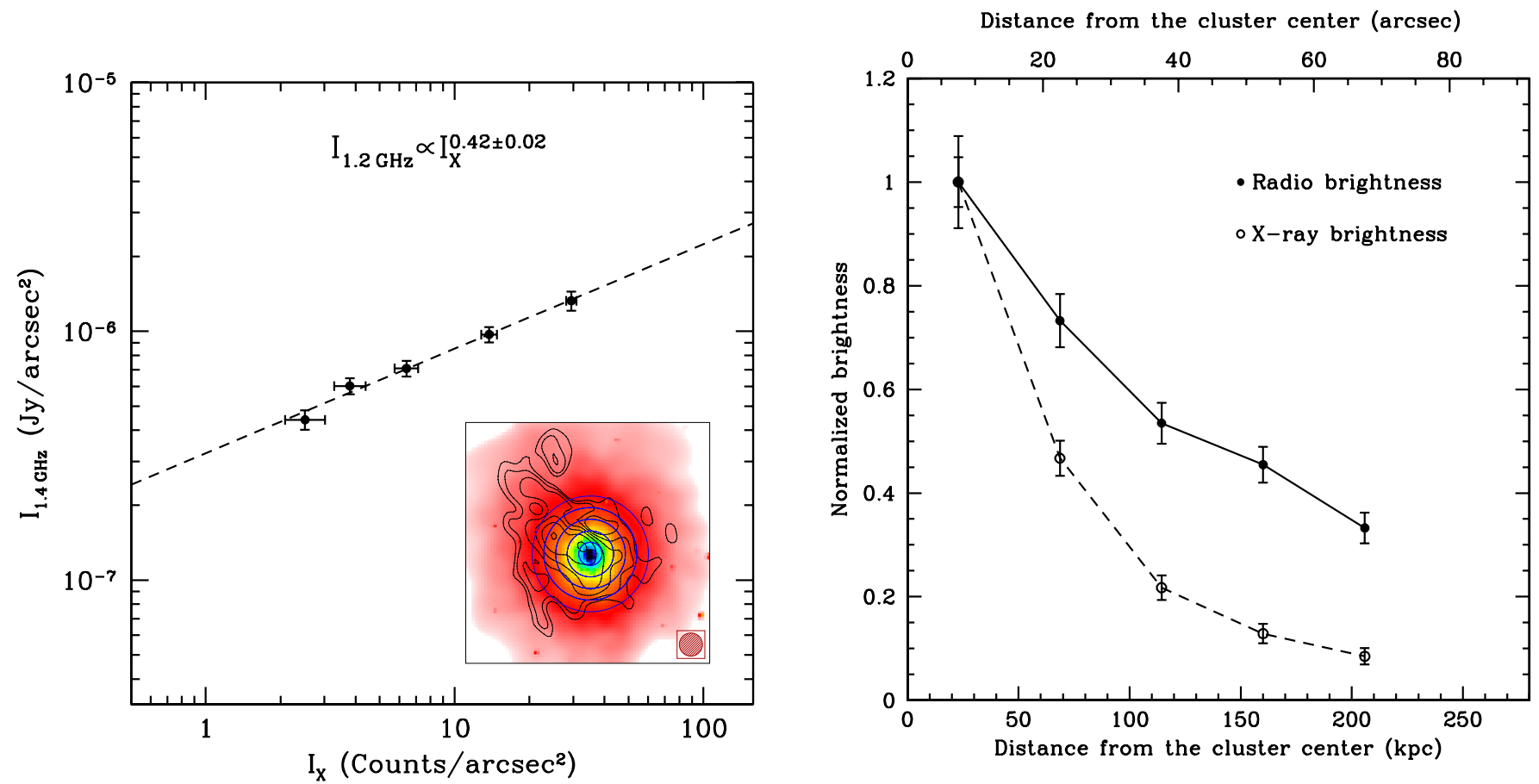

Fig. 6. Left: azimuthal average of the radio brightness at $1.2 \mathrm{GHz}$ versus the Chandra X-ray image in A1689. Each dot represents the average of the emission in an annulus of half beam-width size $\left(15^{\prime \prime}\right)$ centered on the cluster X-ray peak, as shown in the inset. Right: normalized radio and $\mathrm{X}$-ray radial profiles.

\section{Discussion and conclusion}

As a part of an ongoing program aimed at finding new diffuse sources in galaxy clusters, we have investigated the radio emission of the complex galaxy cluster A1689. We analyzed deep radio observations carried out with the VLA at 1.2 and $1.4 \mathrm{GHz}$ in different configurations. To properly map these diffuse sources, high sensitivity to the extended features is needed, along with modest $\left(\sim 20^{\prime \prime}\right)$ angular resolution in order to distinguish a real diffuse source from the blend of unrelated discrete sources. The availability of sensitive radio images at different resolutions revealed that at the center of A1689 a few discrete sources are surrounded by a diffuse low surface brightness emission associated with the ICM.

We studied the morphological and physical radio properties (i.e., length scale, central brightness, average emissivity) of the diffuse emission in A1689 in comparison with that of other halos known in the literature, by fitting their azimuthally averaged brightness profile with an exponential law. The best-fit of the exponential model at $1.2 \mathrm{GHz}$ yields a central brightness of $I_{0}=1.7 \mu \mathrm{Jy} / \operatorname{arcsec}^{2}$ and $r_{\mathrm{e}}=149 \mathrm{kpc}$. From the central brightness and the e-folding radius, we derived an average radio emissivity of $\langle J\rangle=1.7 \times 10^{-42} \mathrm{ergs}^{-1} \mathrm{~cm}^{-3} \mathrm{~Hz}^{-1}$, consistent with that of all the other radio halo clusters known to date. Moreover, we investigated the possible presence of a radio - X-ray correlation in A1689. We find a sublinear slope, which comes from the radial profile of the radio emission being flatter than the X-ray one.

The presence of a radio halo in A1689 strongly supports the cluster merger scenario discussed by Leonard et al. (2011). Detecting a diffuse nonthermal emission in a high-mass, merging structure agrees with the evidence that radio halos are mostly found in massive merging systems.

Acknowledgements. We thank the referee for the very useful comments that helped to improve this paper. This research was partially supported by
PRIN-INAF2009. We acknowledge financial contribution from the agreement ASI-INAF I/009/10/0. We thank Annalisa Bonafede and Roberto Pizzo for useful discussions. V.V. acknowledges the hospitality of the Department of Physics and Astronomy at the University of New Mexico, where part of this work was done. G.B.T. acknowledges support provided by the National Aeronautics and Space Administration through Chandra Award Numbers GO011139X and GO0-11138B issued by the Chandra X-ray Observatory Center, which is operated by the Smithsonian Astrophysical Observatory for and on behalf of the National Aeronautics Space Administration under contract NAS803060. The National Radio Astronomy Observatory (NRAO) is a facility of the National Science Foundation, operated under cooperative agreement by Associated Universities, Inc. Funding for the SDSS and SDSS-II has been provided by the Alfred P. Sloan Foundation, the Participating Institutions, the National Science Foundation, the US Department of Energy, the National Aeronautics and Space Administration, the Japanese Monbukagakusho, the Max Planck Society, and the Higher Education Funding Council for England. The SDSS Web Site is http://www.sdss.org/. This research made use of Montage, funded by the National Aeronautics and Space Administration's Earth Science Technology Office, Computational Technologies Project, under Cooperative Agreement Number NCC5-626 between NASA and the California Institute of Technology. The code is maintained by the NASA/IPAC Infrared Science Archive.

\section{References}

Andersson, K. E., \& Madejski, G. M. 2004, ApJ, 607, 190

Bacchi M., Feretti L., Giovannini G., \& Govoni, F. 2003, A\&A, 400, 465

Bonafede, A., Feretti L., Giovannini G., et al. 2009, A\&A, 503, 707

Broadhurst, T., Takada, M., Umetsu, K., et al. 2005a, ApJ, 619, L143

Broadhurst, T., Benítez N., Coe, D., et al. 2005b, ApJ, 621, 53

Brown, S., \& Rudnick, L. 2011, MNRAS, 412, 2

Brown, S., Duesterhoeft, J., \& Rudnick, L. 2011, ApJ, 727, L25

Brunetti, G., \& Lazarian, A. 2007, MNRAS, 378, 245

Brunetti, G., Venturi, T., Dallacasa, D., et al. 2007, ApJ, 670, L5

Buote, D. A. 2001, ApJ, 553, L15

Burns, J. O., Sulkanen, M. E., Gisler, G. R., \& Perley, R. A. 1992, ApJ, 388, L49 Burns, J. O., Hallman, E. J., Gantner, B., et al. 2008, ApJ, 675, 1125

Cassano, R., Ettori, S., Giacintucci, S., et al. 2010, ApJ, 721, L82

Chen, Y., Reiprich, T. H., Böhringer, H., Ikebe, Y., \& Zhang, Y.-Y. 2007, A\&A, 466, 805

Clowe, D., \& Schneider, P. 2001, A\&A, 379, 384

Coe, D., Benítez, N., Broadhurst, T., \& Moustakas, L. A. 2010, ApJ, 723, 1678 
Colafrancesco, S., 1999, Diffuse Thermal and Relativistic Plasma in Galaxy Clusters, 269

Ensslin, T. A., Biermann, P. L., Klein, U., \& Kohle, S. 1998, A\&A, 332, 395

Feretti, L. 2002, The Universe at Low Radio Frequencies, Pune, India, ed. A. Pramesh Rao, G. Swarup, \& Gopal-Krishna, Proc. IAU Symp., 199, 133

Feretti, L., \& Giovannini, G. 2008, A Pan-Chr. View of Clust. of Galax. and the Large-Scale Struct., 740, 143

Ferrari, C., Govoni, F., Schindler, et al. 2008, Space Sci. Rev., 134, 93

Giovannini, G., \& Feretti, L. 2000, New Astron., 5, 335

Giovannini, G., Bonafede, A., Feretti, L., et al. 2009, A\&A, 507, 1257

Giovannini, G., Feretti, L., Girardi, M., et al. 2011, A\&A, 530, L5

Girardi, M., Fadda, D., Escalera, E., et al. 1997, ApJ, 490, 56

Gitti, M., Brunetti, G., Feretti, L., \& Setti, G. 2004, A\&A, 417, 1

Gitti, M., Ferrari, C., Domainko, W., et al. 2007, A\&A, 470, L25

Govoni, F., Enßlin, T. A., Feretti, L., \& Giovannini, G. 2001, A\&A, 369, 441

Govoni, F., Markevitch, M., Vikhlinin, A., et al. 2004, ApJ, 605, 695

Govoni, F., Murgia M., Markevitch, M., et al. 2009, A\&A, 499, 371

Govoni, F., Murgia, M., Giovannini, G., Vacca, V., \& Bonafede, A. 2011, A\&A, 529, A69

Kawahara, H., Suto, Y., Kitayama, T., et al. 2007, ApJ, 659, 257

Lemze, D., Barkana, R., Broadhurst, T. J., \& Rephaeli, Y. 2008, MNRAS, 386, 1092

Leonard, A., King, L. J., \& Goldberg, D. M. 2011, MNRAS, 413, 789

Liang, H., 1999, Diffuse Thermal and Relativistic Plasma in Galaxy Clusters, 33
Limousin, M., Richard J., Jullo E., et al. 2007, ApJ, 668, 643 Miralda-Escude, J., \& Babul, A. 1995, ApJ, 449, 18

Murgia, M., Govoni, F., Feretti, L., et al. 2004, A\&A, 424, 429

Murgia, M., Govoni, F., Markevitch, M., et al. 2009, A\&A, 499, 679

Murgia, M., Govoni, F., Feretti, L., \& Giovannini, G. 2010, A\&A, 509, A86

Pedlar, A., Ghataure, H. S., Davies, R. D., et al. 1990, MNRAS, 246, 477

Peng, E.-H., Andersson, K., Bautz, M. W., \& Garmire, G. P. 2009, ApJ, 701, 1283

Peres, C. B., Fabian, A. C., Edge, A. C., et al. 1998, MNRAS, 298, 416

Reiprich, T. H., \& Böhringer, H. 2002, ApJ, 567, 716

Riemer-Sørensen, S., Paraficz, D., Ferreira, D. D. M., et al. 2009, ApJ, 693, 1570

Roettiger, K., Stone, J. M., \& Burns, J. O. 1999, ApJ, 518, 594

Rossetti, M., Eckert, D., Cavalleri, B. M., et al. 2011, A\&A, 532, A123

Sarazin, C. L. 2002, Merging Processes in Galaxy Clusters, ed. L. Feretti, I. M.

Gioia, G. Giovannini (Kluwer Academic Publishers, Dordrecht), Astrophys. Space Sci. Lib., 272, 1

Skillman, S. W., O'Shea, B. W., Hallman, E. J., Burns, J. O., \& Norman, M. L. 2008, ApJ, 689, 1063

Skillman, S. W., Hallman, E. J., O'Shea, B. W., et al. 2011, ApJ, 735, 96

Struble, M. F., \& Rood, H. J. 1999, ApJS, 125, 35

Tyson, J. A., Wenk, R. A., \& Valdes, F. 1990, ApJ, 349, L1

Umetsu, K., \& Broadhurst, T. 2008, ApJ, 684, 177

White, D. A., \& Fabian, A. C. 1995, MNRAS, 273, 72

Xu, H., Li, H., Collins, D. C., Li, S., \& Norman, M. L. 2010, ApJ, 725, 2152 